\title{
Decompressive craniectomy in malignant middle cerebral artery infarctions: outcome of 25 cases
}

\author{
Mohamed Ibrahim Refaat ${ }^{*}$ and Omar Youssef Abdallah
}

\begin{abstract}
Background: Large space-occupying middle cerebral artery (MCA) infarctions with signs of elevated intracranial pressure and brain herniation have overall mortality rates of $80 \%$. Several studies have suggested that decompressive surgery reduces mortality and improves outcome of these patients.

Aim: The aim of this study was to evaluate the clinical and radiological outcome of decompressive craniectomy in the setting of malignant MCA infarctions.

Patients and method: This is a prospective study conducted on patients presenting with manifestations of malignant MCA infarction. All cases were operated upon by decompressive craniectomy and duroplasty within $6 \mathrm{~h}$ of presentation or deterioration in Glasgow Coma Scale (GCS). Functional outcome was assessed in terms of mortality and modified Rankin Scale (mRS) for neurologic disability. Radiological outcome was assessed by comparing the midline shift in the preoperative $\mathrm{CT}$ and 24 to $96 \mathrm{~h}$ postoperatively.

Results: Twenty-five patients were included in this study. The mean age was 64.4 years. The mean preoperative GCS score was 8.14. The mean preoperative midline shift was $7.9 \mathrm{~mm}$. All cases showed radiological improvement in the early follow-up period. Good functional outcome based on mRS was achieved in $64 \%$ and poor outcome in $36 \%$ of cases. The overall mortality in this study was $28 \%$.

Conclusion: Early decompressive hemicraniectomy had a proven role in reduction of mortality and improving functional outcome in cases with malignant MCA infarction. Among other factors, timing of surgery and the preoperative clinical condition were factors that affected the final outcome.
\end{abstract}

Keywords: Decompressive craniectomy, Malignant MCA infarction, Functional outcomes modified Rankin Scale, Brain herniation

\section{Introduction}

The term "malignant middle cerebral artery (MCA) infarction" is used to describe a distinct type of cerebral infarctions, characterized by total occlusion of the MCA. These are the most devastating form of acute stroke, with an overall mortality of $80 \%$. They represent $8-15 \%$ of all cerebrovascular strokes. Malignant MCA infarction is characterized by acute massive brain edema, elevated intracranial pressure, and uncal herniation. Malignant infarction may also contain another type showing

\footnotetext{
* Correspondence: mrefaat_77@yahoo.co.uk

Department of Neurosurgery, School of Medicine, Cairo University, Cairo, Egypt
}

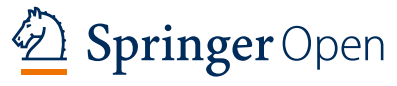

(c) The Author(s). 2018 Open Access This article is distributed under the terms of the Creative Commons Attribution 4.0 International License (http://creativecommons.org/licenses/by/4.0/), which permits unrestricted use, distribution, and reproduction in any medium, provided you give appropriate credit to the original author(s) and the source, provide a link to the Creative Commons license, and indicate if changes were made. artey (ACA) territories [5, 8].

The neurological manifestations of cerebrovascular stroke are more severe in cases of malignant MCA infarctions, including hemiplegia, cranial nerve affections, hemianasthesia, and global aphasia. These signs usually progress to disturbed conscious level and death with 24 to $72 \mathrm{~h}$. Other terms used to describe this condition include "space-occupying MCA infarction," "massive MCA infarction," and "cerebral infarction with swelling" $[3,7]$.

The optimal management protocol for malignant MCA infarction remains controversial, with many studies comparing the outcome of conservative medical treatment alone, or combined with surgical decompression in the 
form of decompressive craniectomy. Decompressive craniectomy has been already proven to be effective in the management of elevated intracranial pressure related to trauma, though its effectiveness in setting of malignant stokes is still under debate. However, several studies report a $40-50 \%$ increase in survival rates when decompressive craniectomy is performed in cases with malignant MCA infarctions [9, 6].

Medical management should take place in a specialized neuro-intensive care unit, with a primary goal of reducing intracranial pressure and maintaining adequate cerebral perfusion pressure, as well as cerebral blood flow. Medical treatment includes hyperventilation, body positioning, hypothermia, brain dehydrating measures (mannitol, hypertonic saline, and corticosteroids), and barbiturate coma [1].

The standard technique for surgical decompression is decompressive hemicraniectomy (or in situ hinged craniotomy) combined with augmentation duroplasty. There is still a controversy concerning the optimal time for surgical intervention and also the cutoff age limit for surgical decompression [9].

The aim of this study was to evaluate the clinical and radiological outcome of decompressive surgery in the setting of malignant MCA infarctions and to study its effect in reducing the overall mortality rates of this fatal condition.

\section{Patients and methods}

This is a prospective clinical study conducted on 25 patients diagnosed to have malignant MCA infarction who presented to the Neurosurgery Department of Cairo University Hospitals. Patients were collected over 6 months (from June to Dec 2016). Cases had a follow-up period ranging from 4 days to 6 months (mean 3 months). We had approval from the local ethical committee concerning aspects of medical research. None of the authors have any competing interest in this research.

Malignant MCA infarction was diagnosed radiologically by the presence of signs of ischemia in more than two thirds of the MCA territory (may be combined with ACA territory) on brain computed tomography (CT) or diffusion-weighted imaging (DWI) whenever available.

Cases diagnosed with malignant MCA infarction showing clinical evidence of ischemic insult, disturbed conscious level (Glasgow Coma Scale $(\mathrm{GCS}) \leq 12$ ), and CT evidence of massive brain edema with midline shift more than $5 \mathrm{~mm}$ (calculated at the level of the septum pellucidum) were included in this study and operated upon by decompressive surgery within $6 \mathrm{~h}$ from deterioration of conscious level. Patients with lost brain stem reflexes, GCS $\leq 4$, intracerebral hemorrhage, and severe hemodynamic instability were excluded from this study (Figs. 1, 2, and 3).
All cases were admitted in the neuro-intensive care unit, were fully resuscitated, full neurological examinations performed, and have routine preoperative investigations done. Surgical intervention was started within $6 \mathrm{~h}$ from admission or from deterioration of conscious level in cases who were primarily managed conservatively. Medical therapies for cerebrovascular stroke and elevated intracranial pressure are started immediately after admission and continued after surgery.

Standard decompressive surgery was performed for all cases according to the following steps: (1) large fronto-tempro-parietal question mark skin incision, (2) a large hemicraniectomy bone flap (minimum $12 \mathrm{~cm}$ diameter), (3) C-shaped opening of the dura, (4) augmentation of the dura either with a pericranial flap or fascia lata graft, (5) the bone flap is placed subcutaneously in the anterior abdomen or kept in situ flail hinged to the temporalis muscle, and (6) the bone flap is planned to be reimplanted 6 months later.

Functional outcome was assessed in terms of mortality and modified Rankin Scale (mRS) for neurologic disability [10]. Good outcome was defined as mRS from 0 to 3 , while poor outcome was defined as mRS from 4 to 6 . Radiological outcome was assessed by comparing the midline shift in the preoperative CT and 24 to $96 \mathrm{~h}$ postoperatively.

\section{Results}

Twenty-five cases with malignant MCA infarctions were included in this study in the period from June to December 2016. In our study, there was 14 males (56\%) and 11 females $(44 \%)$. The age of the cases ranged from 35 to 72 years; the mean age was 64.4 years. Two cases were less than 50 years (8\%), 7 cases between 50 and 60 years (28\%), 15 cases between 60 and 70 years $(60 \%)$, and 1 case above 70 years (4\%). Twenty-three cases had MCA infarctions (92\%), while 2 cases had both MCA and ACA infarctions. Fourteen cases were right sided while 11 cases were having left-sided infarctions.

It was the first ischemic insult in 21 cases (84\%), while it was on top of a previous stoke in 4 cases (16\%). Seventy-two percent of cases were hypertensive, $44 \%$ were diabetic, $36 \%$ had atrial fibrillation, and $20 \%$ had ischemic heart disease. Cases included in this study were selected to have GCS scores more than 4 and less than 13. Most of the cases (56\%) had GCS scores between 7 and 10, 28\% had GCS scores 5 and 6, while 16\% had GCS scores 11 and 12. The mean preoperative GCS score was 8.14.

The study group was selected to have midline shift more than $5 \mathrm{~mm}$. The mean preoperative midline shift was $7.9 \mathrm{~mm}$. Thirty-six percent of cases had preoperative midline shift between 5 and $7.5 \mathrm{~mm}, 60 \%$ had midline shift between 7.5 and $10 \mathrm{~mm}$, while $4 \%$ had midline shift more than $10 \mathrm{~mm}$. 

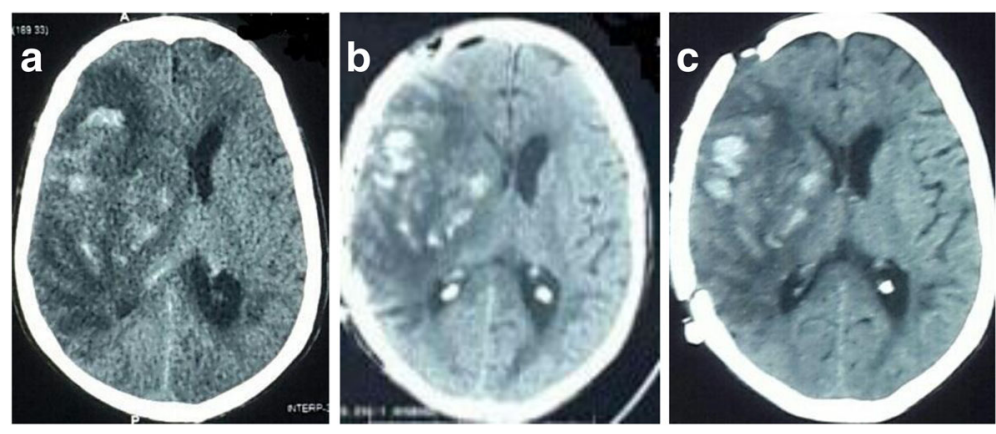

Fig. 1 a 65-year-old male patient with malignant right-sided MCA infarction, preoperative GCS 7/15, and preoperative midline shift $12 \mathrm{~mm}$. b 24 h postoperative $C T$ showing reduction of midline shift. c Delayed postoperative $C T$ showing normalization of midline shift, patient clinically improved and discharged with GCS 15 and mRS 3

All cases were operated upon within $6 \mathrm{~h}$ from the initial presentation in 16 cases $(76 \%)$, while the remaining $24 \%$ of cases were managed conservatively initially as they did not meet our inclusion criteria, but they deteriorated and were included in the study; they were operated upon also within $6 \mathrm{~h}$ from the deterioration. All cases were operated upon by decompressive hemicraniectomy and duroplasty; the bone flap was placed subcutaneously in the anterior abdomen in $76 \%$ of cases or kept in situ flail hinged to the temporalis muscle in $24 \%$ of cases. We did not meet intraoperative complications or mortalities in any of our cases.

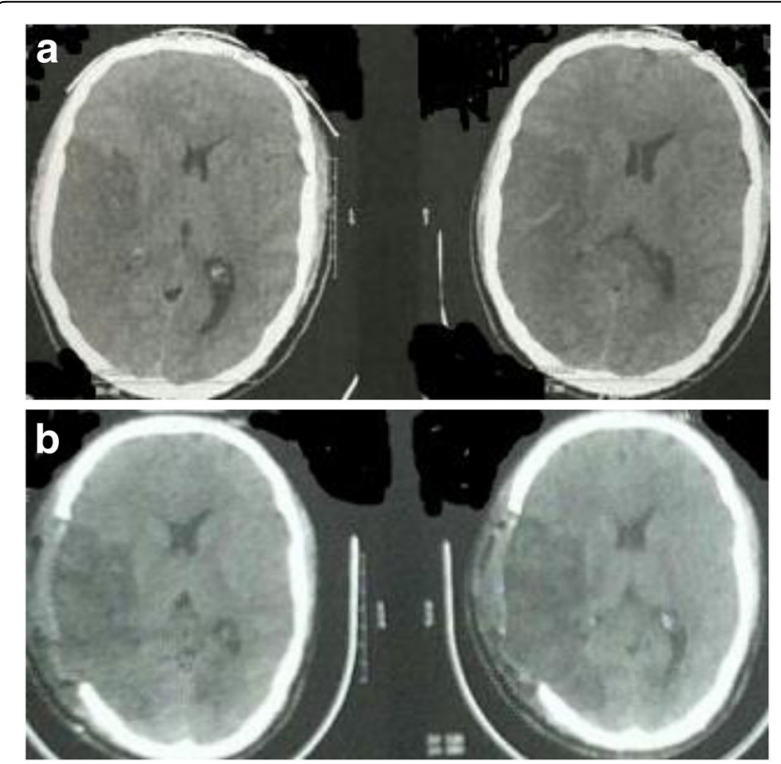

Fig. 2 a 35-year-old male patient with malignant right-sided MCA infarct, preoperative GCS 8/15, and midline shift $6 \mathrm{~mm}$. $\mathbf{b}$ Postoperative $C T$ after 3 days showing resolution of midline shift. Patient was discharged with GCS 15 and mRS 4
All cases showed radiological improvement concerning the midline shift in the follow-up CT scans; the mean postoperative midline shift in the first $24 \mathrm{~h}$ follow-up CT was $4.8 \mathrm{~mm}$. The midline shift resolved in all cases within 7 postoperative days; the mean was 3.6 days. There was 7 mortality cases in this study (28\%): 4 of these cases did not show any clinical improvement after surgical decompression (despite radiologic improvement of midline shift), while the remaining 3 cases showed initial clinical improvement but later on died. Two of the cases (8\%) had hemorrhagic transformation of the stroke after decompression; both were moderate sized and were managed conservatively. ICU-related complications (bed sores, pneumonia, DVT, and sepsis) occurred in 11 cases (44\%) and were fatal in 3 cases (12\%).

Good outcome (mRS 0 to 3 ) was achieved in 16 cases (64\%), while poor outcome (mRS 4 to 6 ) was found in 9 cases (36\%). The mean mRS at the end of the follow-up period was 3.36 .

\section{Discussion}

Malignant MCA infarction is a life-threatening condition caused by complete occlusion of the MCA alone or may be combined with ACA occlusion. It is diagnosed radiologically by the presence of signs of ischemia in more than two thirds of the MCA territory associated with massive brain edema and uncal herniation. Malignant MCA infarction occurs in 10\% of supratentorial strokes. It does not have a certain age group at risk, and clinically worsening usually occurs in the first $24-48 \mathrm{~h}$ [4]. The main suspected cause of death in cases of malignant MCA infarction is thought to be the massive brain edema leading to uncal herniation and brain stem compression.

Conventional therapies for malignant MCA infarctions are directed to reduce the brain edema and elevated intracranial pressure and also to maintain adequate cerebral perfusion to spare the penumbra areas as well as the 


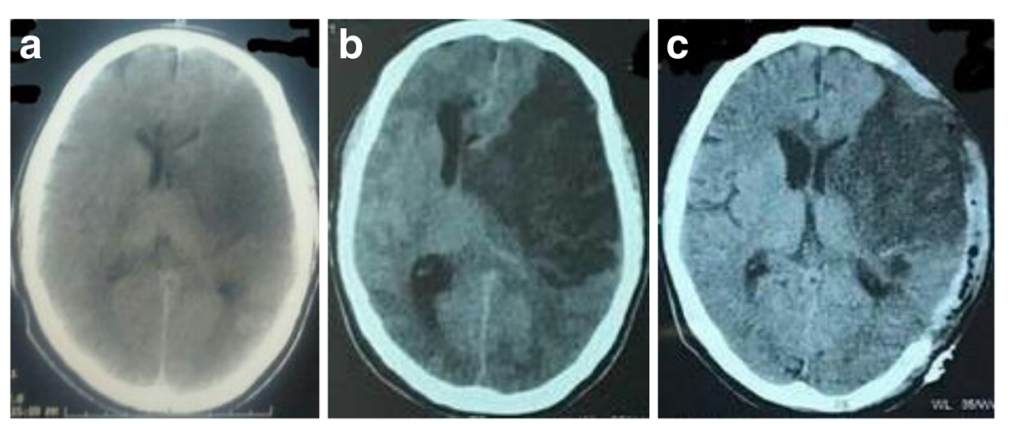

Fig. 3 a 49-year-old male presenting with left MCA infarct, GCS 13/15. b CT after deterioration, GCS 6/15, midline shift 10 mm. c Postoperative CT showing resolution of midline shift, patient was discharged GCS 15 and mRS 4

non-ischemic areas. Non-surgical therapies include positioning, hyperventilation, barbiturate coma, hypothermia, and brain dehydrating therapy (mannitol, hypertonic saline, corticosteroids, glyburide, and glycerol). However, all these measures are not always effective and sometimes elevated intracranial pressure is refractory to this medical therapy and leads to mortality.

Decompressive craniectomy has been proven to be an effective tool for reduction of malignant elevations of intracranial pressure in cases of head trauma and during cranial surgeries. The aim of decompressive surgery is to open the rigid skull converting it to an open box. According to the Monro-Kellie doctrine, increasing the volume of the skull will help to reduce the intracranial pressure. Just removal of the bone flap was proven to reduce ICP by $15 \%$; further reduction, up to $70 \%$, occurs after opening the dura. Elevation of a 12-cm-diameter bone flap will add 86-ml additional volume to the skull [2].

Our study was conducted upon 25 patients with radiologically proven malignant MCA infarcts; all were chosen to have a midline shift more than $5 \mathrm{~mm}$ preoperatively and were also chosen to have GCS scores between 5 and 12. We studied the effect of early decompressive surgery on the clinical outcome and overall mortality rates. One of the authors proved in a previous study that leaving the bone flap hinged flail to the temporalis muscle has the same efficacy of removing it totally, so both techniques were used in this study [13].

Many other researchers have published promising results of early decompressive hemicraniectomy for malignant MCA infarcts. Most of the studies stated that the incidence of permanent major disability or persistent vegetative state remains unchanged but there is a marked reduction in the overall mortality rates and also an increased percentage of cases with a good functional outcome. Three European randomized controlled trials were recently published studying the effect of decompressive surgery for malignant MCA infarcts: the German DESTINY trial, the Dutch HAMLET trial, and the
French DECIMAL trials $[9,6,12]$. Pooled analysis of these studies demonstrated reduction in mortality rates by $49 \%$ and good functional increase by $22 \%$ compared to conservative treatment group.

In our study, the mean age of cases was 64.4 years, $56 \%$ were males, and $86 \%$ of cases had prestoke comorbidities (hypertension, diabetes, ischemic heart disease, and AF). All cases showed radiologic improvement of the midline shift, and the mean resolution time was 3.6 days. We had $28 \%$ mortalities among our cases, favorable functional outcome (mRS 0-3) in 64\% of cases, and poor outcome (mRS 4-6) in $36 \%$ of cases, with a mean mRS 3.36 at the end of the follow-up period. In all our cases, surgical decompression was done early, within $6 \mathrm{~h}$ from presentation or deterioration. We had no surgery-related mortalities or complications; only two cases had hemorrhagic transformation that did not affect the final outcome. Age, preoperative size of midline shift, comorbidities, and side of the infarct did not have a statistically significant effect on the final outcome. The preoperative clinical condition and GCS score were the only factor affecting the final outcome.

These results were supporting several published results, as well as the three main European trials investigating malignant MCA infarcts. The study published by Bansal et al. demonstrated mean mRS 3.33 at discharge [2], and the study published by Raffiq MA showed good functional outcome based on mRS in $48.9 \%$ of patients at 3 months and in $64.4 \%$ of patients at 6 months [11]. Also the pooled analysis of the three European trials showed $43 \%$ of cases had favorable functional outcome and $29 \%$ mortality rate $[9,6,12]$.

\section{Conclusion}

Malignant MCA infarcts, with massive brain edema and uncal herniation, remains as a life-threating condition that has to be instantly managed in order to reduce mortalities and morbidities. Early decompressive hemicraniectomy has been proven to be an effective tool to reduce mortality 
rates and increase probability of favorable functional outcome after malignant MCA infarcts. Factors like patients' age, site of infarct, and preoperative midline shift did not affect the final outcome. Preoperative clinical condition, preoperative GCS scores, and early timing of surgery led to favorable clinical outcome. A study with larger sample size and longer follow-up is recommended for better confirmation of these facts.

\section{Abbreviations}

ACA: Anterior cerebral artery; CT: Computed tomography; DWI: Diffusion weighted images; MCA: Middle cerebral artery; mRS: Modified Rankin Scale

\section{Acknowledgements}

Not applicable.

\section{Funding}

This research was self-funded by the authors. Surgeries were performed in Cairo University Hospital, which is a free public educational hospital.

\section{Availability of data and materials}

All data included in this study is available for sharing, including the master table. It can be requested from the corresponding author upon request.

\section{Authors' contributions}

Both authors participated evenly in this research, and both operated the cases, participated in the data analysis, and writing of the manuscript. Also both authors gave the final approval for this version to be published. The author MIR initiated the idea of this research, designed the research, participated in the data collection and analysis, and revised the final manuscript. The author OYA participated in the data collection and analysis, did the literature review, and wrote the manuscript.

\section{Ethics approval and consent to participate}

We had approval from the local ethical committee concerning aspects of medical research. All cases included in this had signed a consent to participate in this research and to publish the data before being included in the study (signed by first-degree relative in non-fully conscious patients). The ethical committee of the Neurosurgery Department, Cairo University, Egypt, approved this research prior to starting it, May 2016 (reference email: mwkotb@kasralainy.edu.eg, reference phone number: +2-0122-211-8951).

\section{Consent for publication}

Not applicable.

\section{Competing interests}

The authors declare that they have no competing interests.

\section{Publisher's Note}

Springer Nature remains neutral with regard to jurisdictional claims in published maps and institutional affiliations.

Received: 12 December 2017 Accepted: 14 October 2018

Published online: 22 October 2018

\section{References}

1. Ardutzky J, Schwab S. Antiedema therapy in ischemic stroke. Stoke. 2007;38: 3084-94.

2. Bansal $H$, Chaudhary $A$, Singh $A$, et al. Decompressive craniectomy in malignant middle cerebral artery infarct: an institutional experience. Asian J Neurosurg. 2015;10(3):203-6.

3. Cho SY, Oh CW, Bae HJ, et al. The prognostic factors that influence longterm survival in acute large cerebral infarction. J Korean Neurosurg Soc. 2011:49(2):92-6.

4. Fandino J, Keller E, Barth A, et al. Decompressive craniotomy after middle cerebral artery infarction. Retrospective analysis of patients treated in three centers in Switzerland. Swiss Med Wkly. 2004;134:423-9.
5. Gwyn JC, Veenith T. Management of malignant middle cerebral artery infarction. EMJ Neurol. 2015;3(1):57-62.

6. Hofmijer J. Hamlet investigators. Surgical decompression for spaceoccupying cerebral infarction (Hemicraniectomy after Middle Cerebral Artery infarction with Life-Threatening Edema Trail [HAMLET]): a multicenter, open, randomized trial. Lancet Neurol. 2009:8:326-33.

7. Huttner HB, Schwab S. Malignant middle cerebral artery infarction: clinical characteristics, treatment strategies, and future perspectives. Lancet Neurol. 2009:8:949-58.

8. Jae Won YU, Choi J-H, Kim D-H, et al. Outcome following decompressive craniectomy for malignant middle cerebral artery infarction in patients older than 70 years old. J Cerebrovasc Endovasc Neurosurg. 2012;14(2):65-74.

9. Juttler E, DESTINY study group. Decompressive surgery for the treatment of malignant infarction of the middle cerebral artery: a randomized, controlled trial. Stroke. 2007:38:2518-25.

10. Quinn TJ, Dawson J, Walter MR, et al. Exploring the reliability of the modified Rankin scale. Stroke. 2009:40:762.

11. Raffiq MA, Haspani MS, Kandasamy R, Abdullah JM. Decompressive craniectomy for malignant middle cerebral artery infarction: impact on mortality and functional outcome. Surg Neurol Int. 2014;26(5):102.

12. Vahedi K, Vicaut MJ, et al. Sequential-design, multicenter, randomized, controlled trial of early decompressive craniectomy in malignant middle cerebral artery infarction (DECEMIAL Trial). Stoke. 2007;38(9):2506-17.

13. Zaater AM, Refaat MI. Temporalis-based hinge craniotomy: technical modification of decompressive craniectomy in head injury. EJNS. 2014; 29(2):49-52.

\section{Submit your manuscript to a SpringerOpen ${ }^{\circ}$ journal and benefit from:}

- Convenient online submission

- Rigorous peer review

- Open access: articles freely available online

- High visibility within the field

- Retaining the copyright to your article

Submit your next manuscript at $\boldsymbol{\nabla}$ springeropen.com 